Health \& Medicine | Michael Dubina

\title{
Aerosolised medicines may reduce the high risk of coronavirus infection
}

Rapid development of vaccines
and other preventative
measures has been vital in the
response to the COVID-19
pandemic. Dr Michael Dubina,
full member of the Russian
Academy of Sciences, explores
a novel method to reduce
coronavirus infection risk.
His pilot study explored the
effects of an aerosolised
combination medication
in healthcare workers who
had not previously been
infected with the virus. The
findings show that use of
aerosolised medicines may
offer an effective pre-exposure
prophylaxis treatment.

\section{o date, almost 3.5 million deaths have been caused by the
worldwide COVID-19 pandemic} and this figure continues to rise.

COVID-19 is the name given to the disease caused following infection with severe acute respiratory syndrome presentation varies from no symptoms (asymptomatic) to the development of pneumonia, acute respiratory distress syndrome or multi-organ failure.

Multiple vaccines against SARS-CoV-2 infection have been approved for use around the world, and there are many more vaccines still in development. One of the most important population to vaccinate is healthcare workers, who are exposed to the virus daily and are at increased risk of developing COVID-19.

HOW DOES SARS-COV-2

\section{INFECT CELLS?}

Like other coronaviruses, SARS-CoV-2

binds to receptors on cel sus-CoV-2

cell-suface binding allows the virus

to initiate the infection process by

entering the cell and hijacking its

replication machinery to produce more

virus particles. Each virus recognises

specific receptor, and this means that

the number and distribution of these

ability to productively infect a cell.

To infect cells, SARS-CoV-2 uses a

cellular receptor called angiotensin-

converting enzyme 2 (ACE2) allowing

it to gain entry to the cell. ACE2

receptors are found on a variety of cell

types, inclualing cells in the upper and migrate down the respiratory tract and trigger an immune response that can lead to severe respiratory problems and low oxygen levels (hypoxia). ACE2 plays an important role in regulating blod pressure through the production Angiotensin II norm ally causes blood vessels, including those in the lung, to constrict - subsequently causing an increase in blood pressure. If SARS CoV-2 reduces ACE2 activity during cell entry, this may increase angiotensin II. activity in the lungs.

The interactions between SARSCoV-2 and ACE2 are also crucial in determining tissue tropism, defined as the types of tissues the virus can infect and progression to COVID-19 disease.

Dr Michael Dubina, Russian Academy of Sciences, hypothesised that the excessive constriction ability of f thousands of smald al atfect tens blood vessels in the lungs, ultimately causing damage to the small air sacs (alveoli) in the lungs.

He proposed that if the ventilationperfusion functions of the lung periphery could be maintained, the this may prevent the initial step of COVID-19. This is because prolonged constriction changes may make alveoli destruction and inflammation. Therefore early intervention may reduce SARS-CoV-2 infectivity and progression to COVID-19.

\section{PRE-EXPOSURE PROPHYLAXIS} To achieve this, aerosolised medication
known to be beneficial for lung function

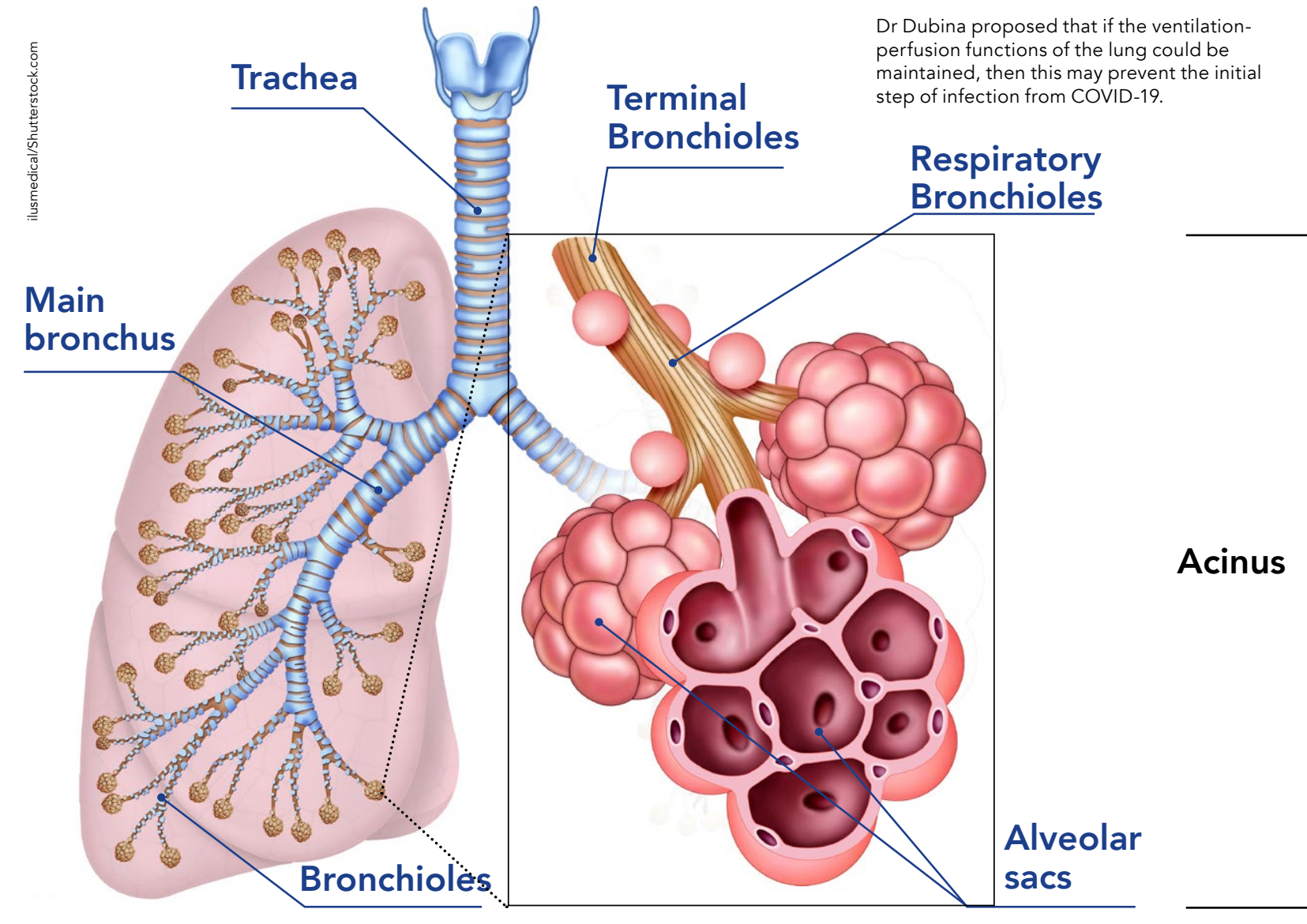

were considered for pre-exposure prophylaxis. In other words, these medications may reduce the risk of infection with SARS-CoV-2 for people who have not yet been infected with the virus as well as prevent the spread of disease in people who have not been exposed to SARS-CoV-2.

Pre-exposure prophylaxis is a Prever reducing the com only HIV A daily pill can be prescribed to HIV-negative adults and adolescents who are at high risk of getting HIV through sex or drug use, and reduces the risk of getting HIV by To date, almost 3.5 million deaths have been caused by the worldwide COVID-19 pandemic and this figure

which was considered earlier in the prophylaxis candidate. However, that it had no effect on coronavirus infection.

\section{AEROSOLISED COMBINATION} MEDICATION (n) health ben fits cound provido exposire prophylaxis against COVID-19

Previous studies have shown that thes medications with greater doses are with no adverse effects reported. Each com no adverse effects reported. Each compont of the medication was lungs and manta abiny to protect the

Glutathione is an antioxidnt that is capable of proxectint molecule damage by highly recting cells fom (n) pre formed as by-products mal oxygen metabolism

\section{continues to rise.}

At present, there is no known agent that can be administered before exposure to SARS-CoV-2 that prevents infection, although several clinical trials are looking at possible therapies. One example of this is used as an anti-malarial drug risk of SARS-CoV-2 infection due to delivering care to patients with confirmed COVID-19.

An aerosolised combination medication (ACM) was used in the trial. This is inosine and potassium chloride. but can be harmful if uncontrolled, a scenario that may

Glutathione is already used successfully in some patients with

cystic fibrosis to help their lung function and has already been used in two cases of COVD-19 to improve COVD-19 elated pneumonia.

Inosine is indirectly used by cells to make ATP, their main energy source 
Schematic diagram of the renin-angiotensin system, proposed SARS-CoV-2 action in acute lung failure, and its prevention with aerosolised medicines

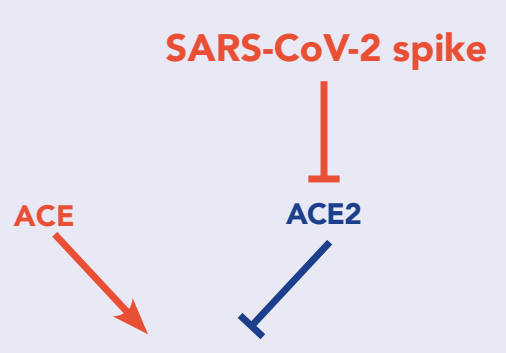

Angiotensin II

$$
\begin{gathered}
\text { Aerosolised } \\
\text { medicines }
\end{gathered} \text { (1) }
$$

Contraction of smooth muscle cells in sma blood vessels and terminal bronchioles

$$
\checkmark
$$

Hypoxia and cellular energy deficiency

in respiratory system

$\checkmark$

Diffuse alveolar damage

$$
\forall
$$

Lung injury

Early intervention may reduce SARS-CoV-2 infectivity and progression to COVID-19 disease.
(Modified from Kuba K. et al. Nature Medicine, 2005, fig. 2a).

Dr Dubina suggests that direct aerosol delivery of appropriate medications may maintain lung function and thus protect from COVID-19.

\section{possible anti-1
the lung.}

Finally, potassium chloride was selected due to its basic physiological ability to dilate blood vessels and small bronchi.

\section{PILOT STUDY OF}

HEALTHCARE WORKERS The study, recently published in BMC Infection Diseases, recruited 99 healthcare workers who had not previously been exposed to SARSCoV-2. The participants received ACM via a hand help nebulsser for 14 days, inssions per day Interestingly the in the control group. Mild short-term

\section{. Behind the Research}

\section{Dr Michael Dubina}

E: michael.dubina@gmail.com

adverse reactions were observed in five

Dubina concludes that direct aerosol delivery of appropriate medications that this is due to prevention of ventilation-perfusion abnormalities in the peripheral area of lung due to a dysregulated balance between ACE2 and angiotensin II, which is induced by SARS-CoV-2 infection.

\section{CONCLUSIONS} is that it was carried out in a single centre only. Due to ethical issues there was no control group to compare to healthcare workers who received an aerosolised intervention that did not contain the medications. (It was decided by local ethics committee that it was not ethical to treat any individuals at high risk for SARS-CoV-2 infection with a placebo during the COVID-19 pandemic). It would also about the participants, for example were they more likely to avoid contact with other people outside work as they were involved in a research study and thus had a reduced risk of infection? Participants were not requested to avoid contact with other people outside the study].

The results of the pilot are promising however, and the next steps may prophylaxis. Dr Dubina aims to do further studies to look at the effect of pre-exposure prophylaxis in different populations at different locations, through a randomised trial, to include placebo treatment alongside ACM.

The findings from this pilot study suggest that ACM may be used to help reduce the risk of SARS-CoV-2 and to progress other research into more effective treatments for COVID-19. Notably, the results also highlight mechanisms and pathways that could be targeted to re
One of the limitations of the study be interesting to collect further data .

\section{Research Objectives} initial step of the infection process from severe acute

\section{Detail}

Address

Uussian Acadeny of Sciences, 14 Leninsky Prospe

Michael Dubina studied medicine at I.P. Pavlov First State Medical University, St. Petersburg, Russia. He received his PhD there in 1998 and DMSc (pathophysiology) in 2004. He completed his Alma mater; Zh.I. Alferov National Academic University Petersburg, Russia; International Agency for Research on Cancer WHO, Lyon, France. He has been honored for outstanding research achievements with D.W. Lubbers award, UNESCO medal and others. He was elected ful member (academician) of Russian Academy of Sciences in 2016

Funding

This study was funded from personal charity financing

Collaborators

Anastasia Taraskina, PhD; Natalia syanov, DMSc, at the (MMU), St. Petersburg, Russia.

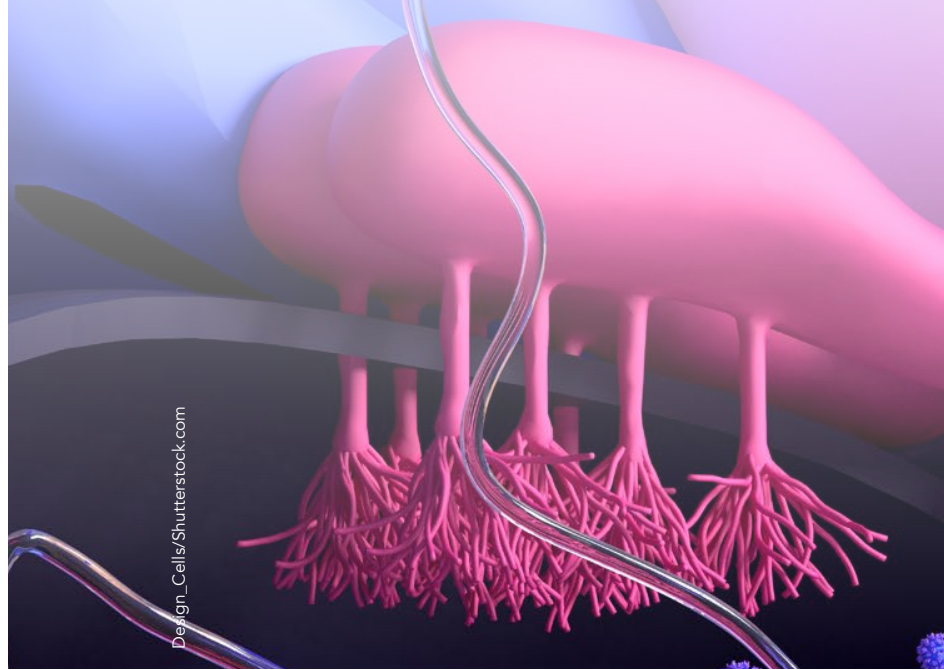

Michael Dubina is developing an aerosol to prevent the respiratory syndrome coronavirus-2 (SARS-CoV-2).

\section{References}

Dubina, M.A, Gomonova, VV, Taraskina, A E Vasilyeva, N.V. \& Sayganov, S.A. (2021). Pathogenesis-based preexposure prophylaxis associated with a low risk of SARS-COV-2 infection in healthcare workers at a designated COVID-19 hospital: a pilot study. BMC Infectious Diseases. 21:536 https://doi.org/10.1186/ D

\section{Personal Response}

What are the long-term effects of frequent administration of ACMs to reduce SARS-CoV-2 infection and does protection wane when ACM is continue inhaling ACM multiple times a day? II Each component of the medication is a simpl II Each component of the medication is a simple biological substance or ion, produced in the lungs to for other physiological functions, for example). Thus, they are not harmful at low doses even during long-term inhalation. In accordance with the results of the pilot study, minimal protection wanes when ACM is stopped point equates to the term of SARS-CoV-2 detection after the end of ACM treatment in only one participant of the study. Maximum protection wanes after ACM is stopped, and may depend on the intrinsic secretory
level of these substances in the lung and could be level of these subs 